\title{
Extremum Seeking-based tracking for Unknown Systems with Unknown Control Directions
}

\author{
Alexander Scheinker, Miroslav Krstic
}

\begin{abstract}
We develop controllers which perform trajectory tracking for a class of unknown linear and nonlinear systems. In the linear case we work under the assumption that the timevarying input vector, which is otherwise unknown, satisfies a persistency of excitation condition over a sufficiently short window. In the multi-input nonlinear case the square of the unknown time-varying input matrix has a known lower bound. Our design does not guarantee perfect regulation to the desired trajectory, but ensures semiglobal uniform ultimate boundedness of the error system and prevents large overshoots even when the initial estimate of the control direction is wrong. The stability analysis is inspired by the approach in a recent work by Dürr, Stankovic, and Johansson, which combines a Lie bracket second-order averaging result of Gurvits and $\mathrm{Li}$ with an extension of the perturbation theory semiglobal practical stability result of Moreau and Aeyels.
\end{abstract}

\section{INTRODUCTION}

a) Motivation: The problem of model-independent semiglobal exponential practical stabilization for any linear time-varying single-input system as well as a class of nonlinear systems was recently solved by the authors [1]. A natural extension of that work was to develop controllers for trajectory tracking which is notoriously difficult in the case of unknown and changing control gain direction.

b) Results of the paper: In this paper we extend the stability results of [1] to ultimately bounded trajectory tracking with a design based on extremum seeking [6], [9]. Our design is inspired by a recent extremum seeking design by Dürr et al [2], where an innovative combination of certain Lie bracket-based second-order averaging results by Gurvits and $\mathrm{Li}$ [3], [4] was combined with results of Moreau and Aeyels [7].

The focus in this paper is on extending the stability results of [1] to trajectory tracking for LTV and nonlinear systems of the form

$$
\dot{x}=A(t) x+B(t) u \text { and } \dot{x}=f(x, t)+G(x, t) u,
$$

for a trajectory $r(t)$ having known magnitude and rate of change bounds. We define the error vector $e(t)=x(t)-$ $r(t)$ and in the linear case, we use a nonlinear time-varying

Alexander Scheinker is a staff member at Los Alamos National Laboratory and in the Doctoral Program (Aerospace and Mechanical) at University of California at San Diego, La Jolla, CA 92093-0411, USA, ascheink@ucsd.edu.

Miroslav Krstic is with the Department of Mechanical and Aerospace Engineering, University of California, San Diego, La Jolla, CA 92093-0411, USA, krstic@ucsd.edu.

This research was supported by Los Alamos National Laboratory and UCSD control law of the form

$$
u=\alpha \sqrt{\omega} \cos (\omega t)-k \sqrt{\omega} \sin (\omega t)|e|^{2},
$$

and in the nonlinear case a control law of the form

$$
u_{i}=\alpha \sqrt{\omega \omega_{i}^{\prime}} \cos \left(\omega \omega_{i}^{\prime} t\right)-k \sqrt{\omega \omega_{i}^{\prime}} \sin \left(\omega \omega_{i}^{\prime} t\right) V(e),
$$

with distinct $\omega_{i}^{\prime}$. We then choose $V(e)$ and gain $k \alpha>0$ sufficiently large to stabilize the linear Lie bracket average error system

$$
\dot{\bar{e}}=\left[A(t)-k \alpha B(t) B^{T}(t)\right] \bar{e}+A(t) r-\dot{r},
$$

or the nonlinear Lie bracket average system

$$
\dot{\bar{e}}=f(\bar{e}, t)-k \alpha \gamma G(\bar{e}, t) G^{T}(\bar{e}, t)\left(\frac{\partial V}{\partial \bar{e}}\right)^{T}-\dot{r},
$$

to within a $\delta$-ball of the origin. In the linear case we assume a persistency of excitation condition on the vector-valued function $B(t)$ and in the nonlinear case we assume that $G(e, t) G^{T}(e, t)>0$. In both cases, following the stabilization of the averaged systems $\omega$ is chosen sufficiently large to satisfy the requirements of the Lie bracket averaging theorem of Gurvits and $\mathrm{Li} \mathrm{[3],} \mathrm{[4]} \mathrm{and} \mathrm{of} \mathrm{a} \mathrm{semiglobal} \mathrm{uniform}$ ultimate boundedness theorem that is a slight modification of the results of Moreau and Aeyels [7].

In the linear case we can not only handle unknown signs of the high frequency gains, but also signs that change with time, allowing $B(t)$ to go through zero. The extremum seeking control scheme is by design operating on a faster time scale than the dynamics in the system's coefficients, and the system's behavior, as estimated by an averaged system, does not depend on the control coefficients' signs. In both cases, we accomplish tracking to within a $\delta$-ball of the desired trajectory. Apart from the limited effort in [10], this paper provides the first results making extremum seeking to tracking for unstable plants.

c) Organization: In Section II we state definitions of stability used throughout the rest of the paper and provide a review of a Lie bracket averaging result of Gurvits and $\mathrm{Li}$ [3], [4] in order to derive an averaged system of our original system. We then use a slight modification of the result of Moreau and Aeyels [7] to show that semiglobal uniform ultimate boundedness of the averaged system implies stability of the original system to within the same $\delta$-ball. In Section III we extend our stability results from [1] to that of trajectory tracking for linear and nonlinear systems. In Section IV we demonstrate the controller's ability to track a trajectory with 
an unstable, 2-dimensional, linear time-varying system.

\section{BACKGROUND ON LIE BRACKET AVERAGING AND} Semiglobal PRACTICAL Ultimate Boundedness

The Lie bracket averaging results of Gurvits and Li [3], [4] apply to systems of the form:

$$
\dot{x}=\sum_{i=1}^{m} b_{i}(x) u_{i}^{\epsilon}(t, \theta), \quad u_{i}^{\epsilon}(t, \theta)=\bar{u}_{i}(t)+\frac{1}{\sqrt{\epsilon}} \hat{u}_{i}(t, \theta),
$$

where $\hat{u}_{i}(t, \theta)$ is $T$-periodic in $\theta=\frac{t}{\epsilon}, T \in(0, \infty)$ and has zero average, $\int_{0}^{T} \hat{u}_{i}(t, \theta) d \theta=0$. Along with the system (5), the following approximation model is considered:

$\dot{z}=\sum_{i}^{m} b_{i}(z) \bar{u}_{i}(t)+\frac{1}{T} \sum_{i<j}\left[b_{i}, b_{j}\right](z) \nu_{i, j}(t), \quad z(0)=x(0)$,

where

$$
\nu_{i, j}(t)=\int_{0}^{T} \int_{0}^{\theta} \hat{u}_{i}(t, \tau) \hat{u}_{j}(t, \theta) d \tau d \theta .
$$

Noting that a $T$-periodic function is also $n T$-periodic, if we replace $T$ with $n T, n \in \mathbb{N}$ and apply the arguments of the original proof ([3], [4]) as well as [13, Lemma2], the systems (5) and (6) then satisfy the following approximation result.

Lemma 1: ([3], [4], [12], [13]) For any period $T>0$ and $n \in \mathbb{N}$, there exists $\epsilon^{\star}$ such that for all $\epsilon \in\left(0, \epsilon^{\star}\right)$, the trajectory of system (5) is within a $\Delta(n T, \epsilon)$-distance of the solution of system (6), namely,

$$
\max _{t \in[0, n T]}|x(t)-z(t)|=\|x-z\|_{C[0, n T]} \leq \Delta(n T, \epsilon),
$$

where $\Delta(n T, \epsilon) \rightarrow 0$ as $\epsilon \rightarrow 0$.

Before we can take advantage of these averaging results we make the following definitions similarly to Moreau and Aeyels [7]. In what follows, given a system

$$
\dot{x}=f(t, x),
$$

$\psi\left(t, t_{0}, x_{0}\right)$ denotes the solution of (9) which passes through the point $x_{0}$ at time $t_{0}$.

Definition 1: Global Uniform Ultimate Boundedness with ultimate bound $\delta(\delta$-GUUB): For $\delta \geq 0$ the origin of (9) is said to be $\delta$-GUUB if it satisfies the following three conditions: $\delta$-Uniform Stability: For every $c_{2} \in(\delta, \infty)$ there exists $c_{1} \in(0, \infty)$ such that for all $t_{0} \in \mathbb{R}$ and for all $x_{0} \in \mathbb{R}^{n}$ with $\left\|x_{0}\right\|<c_{1},\left\|\psi\left(t, t_{0}, x_{0}\right)\right\|<c_{2} \quad \forall t \in\left[t_{0}, \infty\right)$. $\delta$-Uniform Ultimate Boundedness: For every $c_{1} \in(0, \infty)$ there exists $c_{2} \in(\delta, \infty)$ such that for all $t_{0} \in \mathbb{R}$ and for all $x_{0} \in \mathbb{R}^{n}$ with $\left\|x_{0}\right\|<c_{1},\left\|\psi\left(t, t_{0}, x_{0}\right)\right\|<c_{2} \quad \forall t \in\left[t_{0}, \infty\right)$. $\delta$-Global Uniform Attractivity: For all $c_{1}, c_{2} \in(\delta, \infty)$ there exists $\bar{T} \in(0, \infty)$ such that for all $t_{0} \in \mathbb{R}$ and for all $x_{0} \in$ $\mathbb{R}^{n}$ with $\left\|x_{0}\right\|<c_{1},\left\|\psi\left(t, t_{0}, x_{0}\right)\right\|<c_{2} \quad \forall t \in\left[t_{0}+\bar{T}, \infty\right)$.

In conjunction with (9), we consider systems of the form

$$
\dot{x}=f^{\epsilon}(t, x)
$$

whose trajectories are denoted as $\phi^{\epsilon}\left(t, t_{0}, x_{0}\right)$.

Definition 2: Converging Trajectories Property: The systems (9) and (10) are said to satisfy the converging tra- jectories property if for every $\hat{T} \in(0, \infty)$ and compact set $K \subset \mathbb{R}^{n}$ satisfying $\left\{\left(t, t_{0}, x_{0}\right) \in \mathbb{R} \times \mathbb{R} \times \mathbb{R}^{n}: t \in\right.$ $\left.\left[t_{0}, t_{0}+\hat{T}\right], x_{0} \in K\right\} \subset \operatorname{Dom} \psi$, for every $d \in(0, \infty)$ there exists $\epsilon^{\star}$ such that for all $t_{0} \in \mathbb{R}$, for all $x_{0} \in K$ and for all $\epsilon \in\left(0, \epsilon^{\star}\right)$,

$$
\left\|\phi^{\epsilon}\left(t, t_{0}, x_{0}\right)-\psi\left(t, t_{0}, x_{0}\right)\right\|<d, \quad \forall t \in\left[t_{0}, t_{0}+\hat{T}\right] .
$$
(10)

We then define the following form of stability for system

Definition 3: $\epsilon$-Semiglobal Practical Uniform Ultimate Boundedness with ultimate bound $\delta((\epsilon, \delta)-S P U U B)$ : The origin of $(10)$ is said to be $(\epsilon, \delta)$-SPUUB if it satisfies the following three conditions: $(\epsilon, \delta)$-Uniform Stability: For every $c_{2} \in(\delta, \infty)$ there exists $c_{1} \in(0, \infty)$ and $\hat{\epsilon} \in(0, \infty)$ such that for all $t_{0} \in \mathbb{R}$ and for all $x_{0} \in \mathbb{R}^{n}$ with $\left\|x_{0}\right\|<c_{1}$ and for all $\epsilon \in(0, \hat{\epsilon}),\left\|\phi^{\epsilon}\left(t, t_{0}, x_{0}\right)\right\|<c_{2} \quad \forall t \in\left[t_{0}, \infty\right)$. $(\epsilon, \delta)$-Uniform Ultimate Boundedness: For every $c_{1} \in(0, \infty)$ there exists $c_{2} \in(\delta, \infty)$ and $\hat{\epsilon} \in(0, \infty)$ such that for all $t_{0} \in \mathbb{R}$ and for all $x_{0} \in \mathbb{R}^{n}$ with $\left\|x_{0}\right\|<c_{1}$ and for all $\epsilon \in(0, \hat{\epsilon}),\left\|\phi^{\epsilon}\left(t, t_{0}, x_{0}\right)\right\|<c_{2} \quad \forall t \in\left[t_{0}, \infty\right)$. $(\epsilon, \delta)$ Global Uniform Attractivity: For all $c_{1}, c_{2} \in(\delta, \infty)$ there exists $\bar{T} \in(0, \infty)$ and $\hat{\epsilon} \in(0, \infty)$ such that for all $t_{0} \in \mathbb{R}$ and for all $x_{0} \in \mathbb{R}^{n}$ with $\left\|x_{0}\right\|<c_{1}$ and for all $\epsilon \in(0, \hat{\epsilon})$, $\left\|\phi^{\epsilon}\left(t, t_{0}, x_{0}\right)\right\|<c_{2} \quad \forall t \in\left[t_{0}+\bar{T}, \infty\right)$.

With these definitions the following modification of the result of Moreau and Aeyels [7] is used in the analysis that follows.

Theorem 1: ([7]) If systems (10) and (9) satisfy the converging trajectories property and if the origin is a $\delta$-GUUB point of (9), then the origin of (10) is $(\epsilon, \delta)$-SPUUB.

Proof: Given any $\hat{T}>0$ we can always choose $n T>\hat{T}$ and therefore by Lemma 1 the solution of system (6) satisfies the converging trajectories property relative to the solution of system (5). The rest of the proof is a slight modification of the proof found in Moreau and Aeyels [7], with a lower bound of $\delta$ instead of 0 on the choices of $c_{2}, b_{2}$ and $c_{3}$, with details available from the author.

\section{TRAJECTORY TRACKING}

We consider tracking for a trajectory $r(t)$ satisfying the bounds $|r(s)|<r_{\star}$ and $|\dot{r}(s)|<\rho_{\star} \forall s \geq T$, where we define $e(t)=x(t)-r(t)$, choose any $\delta>0$ and perform our analysis for $|e| \geq \delta$. We state our results for the simple one dimensional linear case first without proof.

Lemma 2: Consider the system

$$
\dot{x}=a(t) x+b(t) u,
$$

and let there exist $\triangle>0, \beta_{0}>0, a_{\star}>0$, and $T>0$ such that $\forall s \geq T, a(t)$ and $b(t)$ satisfy

$$
\frac{1}{\triangle} \int_{s}^{s+\triangle} b^{2}(\tau) d \tau \geq \beta_{0},|a(s)|<a_{\star} .
$$

Given the error system

$$
\begin{aligned}
& \dot{e}=a(t) e+b(t) u+a(t) r-\dot{r} \\
& u=\alpha \sqrt{\omega} \cos (\omega t)-k \sqrt{\omega} \sin (\omega t) e^{2},
\end{aligned}
$$


if

$$
k \alpha>\frac{a_{\star}\left(r_{\star}+\delta\right)+\rho_{\star}}{\delta \beta_{0}}
$$

then the origin of system (14), (15) is $\left(\frac{1}{\omega}, \delta\right)$-SPUUB.

Before we state our n-dimensional linear results we introduce the notation

$$
\langle Z\rangle_{\Delta}(s) \triangleq \frac{1}{\Delta} \int_{s}^{s+\Delta} Z(\tau) d \tau
$$

for $Z: \mathbb{R} \rightarrow \mathbb{R}$, and note that, for any column vector $B$, $B B^{T} \leq|B|^{2} I$.

Theorem 2: Consider the system

$$
\dot{x}=A(t) x+B(t) u,
$$

where $x \in \mathbb{R}^{n}, A \in \mathbb{R}^{n \times n}, B \in \mathbb{R}^{n}, u \in \mathbb{R}$, and let there exist $\Delta>0, b_{\star} \geq \beta_{0}>0, a_{\star} \geq 0$, and $T \geq 0$ such that $A(t)$ and $B(t)$ satisfy

$$
\begin{aligned}
& \frac{1}{\Delta} \int_{s}^{s+\Delta} B(\tau) B^{T}(\tau) d \tau \geq \beta_{0} I, \forall s \geq T \\
& \left\langle|B|^{2}\right\rangle_{\Delta}(s) \leq b_{\star}, \forall s \geq 0, \\
& \left\langle|A|^{2}\right\rangle_{\Delta}(s) \leq a_{\star}, \forall s \geq 0 .
\end{aligned}
$$

We consider the error system

$$
\begin{aligned}
& \dot{e}=A(t) e+B(t) u+A(t) r-\dot{r}, \\
& u=\alpha \sqrt{\omega} \cos (\omega t)-k \sqrt{\omega} \sin (\omega t)|e|^{2} .
\end{aligned}
$$

The origin of system (22), (23) is $\left(\frac{1}{\omega}, \delta\right)$-SPUUB with average decay rate

$$
R=\frac{1}{2 \Delta}\left[\ln \left(\frac{1}{\gamma_{3}}\right)-a_{\star} \gamma_{2}\right]-\frac{\sqrt{a_{\star}} \delta+r_{\star}+\rho_{\star}}{\delta}>0,
$$

where

$$
\begin{aligned}
\gamma & =1-\frac{k \alpha \Delta \beta_{0}}{1+2 k^{2} \alpha^{2} \Delta^{2} b_{\star}^{2}}>0, \\
\gamma_{2} & =\frac{4 k \alpha \Delta^{3} b_{\star}}{1+2 k^{2} \alpha^{2} \Delta^{2} b_{\star}^{2}}, \\
\gamma_{3} & =\frac{2 \Delta b_{\star}\left(\sqrt{a_{\star}} r_{\star}+\rho_{\star}\right)(1-\gamma)}{\delta^{2} \beta_{0}}+\gamma
\end{aligned}
$$

under either of the two conditions:

(i) Given $k \alpha>0$ and $\Delta>0, a_{\star}$ is in the interval $\left(0, \bar{a}_{\star}\right)$ where $\bar{a}_{\star}$ satisfies

$$
\gamma_{3}\left(\bar{a}_{\star}\right)=e^{-\left(2 \Delta\left[\sqrt{\bar{a}_{\star}}\left(1+\frac{r_{\star}}{\delta}\right)+\frac{\rho_{\star}}{\delta}\right]+\bar{a}_{\star} \gamma_{2}\right)} .
$$

(ii) For a given $a_{\star}$, the window $\Delta$ satisfies $\Delta \in$ $\left(0, \min \left\{\bar{\Delta}_{1}, \bar{\Delta}_{2}\right\}\right)$, where $\bar{\Delta}_{1}$ and $\bar{\Delta}_{2}$ satisfy

$$
\begin{aligned}
0= & \bar{\Delta}_{1}^{2} \sqrt{2} a_{\star}+\bar{\Delta}_{1} 2\left(\sqrt{a_{\star}}\left[1+\frac{r_{\star}}{\delta}\right]+\frac{\rho_{\star}}{\delta}\right) \\
& +\ln \left(1+\frac{\beta_{0}}{2 \sqrt{2} b_{\star}}\left[\frac{2 \bar{\Delta}_{1} b_{\star}\left(\sqrt{a_{\star}} r_{\star}+\rho_{\star}\right)}{\delta^{2} \beta_{0}}-1\right]\right), \\
0= & \beta_{0} \delta^{2}-2 \bar{\Delta}_{2} b_{\star}\left(\sqrt{a_{\star}} r_{\star}+\rho_{\star}\right) \\
& -2 \sqrt{2} b_{\star} \delta^{2}\left[1-e^{-2 \bar{\Delta}_{2}\left(\frac{\sqrt{a_{\star}} \delta+r_{\star}+\rho_{\star}}{\delta}+\frac{a_{\star}}{b_{\star}}\right)}\right],
\end{aligned}
$$

and $k \alpha>1$ is selected such that

$$
k \alpha \in\left(\frac{1}{2 \sqrt{2} \Delta b_{\star}}, \frac{1}{2 \sqrt{2} \Delta b_{\star}}+M_{k}\left(a_{\star}, b_{\star}, \beta_{0}, \Delta\right)\right),
$$

where

$$
M_{k}=\frac{\sqrt{\beta_{0}^{2}\left[\frac{1-\gamma_{3}}{1-\gamma}\right]^{2}-8 b_{\star}^{2}\left[1-e^{\left.-2 \Delta\left(\frac{\sqrt{a_{\star}} \delta+r_{\star}+\rho_{\star}}{\delta}+\frac{a_{\star}}{b_{\star}}\right)\right]^{2}}\right.}}{2 \sqrt{2} b_{\star}\left[1-e^{\left.-2 \Delta\left(\frac{\sqrt{a_{\star} \delta+r_{\star}+\rho_{\star}}}{\delta}+\frac{a_{\star}}{b_{\star}}\right)\right]}\right.} .
$$

Remark 1: Theorem 2(i) is a robustness result. For any $k \alpha>0$, the controller (23) allows some perturbation $A(t) x$ in the system (18), as long as the mean of $A(t)$ is sufficiently small, as quantified by (28). Theorem 2(ii) is a design result. If an accuracy goal $\delta>0$ is chosen such that the window $\Delta$ is small enough, as quantified by (29), (30) then it is possible to choose $k \alpha$ in some range, as quantified by (31) such that $x(t)$ exponentially converges to a trajectory that is within a $\delta$-ball of the trajectory $r(t)$.

Remark 2: Parts of this proof use steps developed in the proof of Theorem 4.3 .2 (iii) in the second half of Section 4.8.3 in [5].

Proof: The Lie bracket averaged closed loop error system (22), (23) is

$$
\dot{\bar{e}}=\left[A(t)-k \alpha B(t) B^{T}(t)\right] \bar{e}+A(t) r-\dot{r} .
$$

With the Lyapunov function candidate $V(\bar{e})=\frac{|\bar{e}|^{2}}{2}$ we get

$$
\dot{V}(\bar{e})=\bar{e}^{T} A(t) \bar{e}-k \alpha \bar{e}^{T} B(t) B^{T}(t) \bar{e}+\bar{e}^{T} A(t) r-\bar{e}^{T} \dot{r} .
$$

Therefore, for any $s \geq T$ we have

$$
\begin{aligned}
& V(s+\Delta)=V(s)-\underbrace{k \alpha \int_{s}^{s+\Delta}\left|\bar{e}^{T}(\tau) B(\tau)\right|^{2} d \tau}_{I_{2}} \\
& +\underbrace{\int_{s}^{s+\Delta} \bar{e}^{T}(\tau) A(\tau) \bar{e}(\tau) d \tau}_{I_{3}}+\underbrace{\int_{s}^{s+\Delta} \bar{e}^{T}(\tau) A(\tau) r(\tau) d \tau}_{I_{4}} \\
& -\underbrace{\int_{s}^{s+\Delta} \bar{e}^{T}(\tau) \dot{r}(\tau) d \tau}_{s} .
\end{aligned}
$$

Applying analysis similar to [11] we get

$$
\begin{aligned}
I_{1} \leq & \frac{-k \alpha \Delta \beta_{0} V(\bar{x})}{1+2 k^{2} \alpha^{2} \Delta^{2} b_{\star}^{2}} \\
& +\frac{k \alpha b_{\star} \Delta^{2}\left(\sqrt{a_{\star}} r_{\star}+\rho_{\star}+4 a_{\star} \int_{s}^{s+\Delta} V(\tau) d \tau\right)}{1+2 k^{2} \alpha^{2} \Delta^{2} b_{\star}^{2}}
\end{aligned}
$$

and

$$
I_{2} \leq 2 \int_{s}^{s+\Delta}|A(\tau)| V(\tau) d \tau
$$

The term $I_{3}$ satisfies the bound

$$
\left|I_{3}\right| \leq \int_{s}^{s+\Delta}\left|\bar{e}^{T}(\tau)\right|^{2} \frac{1}{\left|\bar{e}^{T}(\tau)\right|}|A(\tau)||r(\tau)| d \tau,
$$


which is, for all $|e|>\delta$ and taking into account the bound on $|r(t)|$, bounded by

$$
\left|I_{3}\right| \leq \frac{r_{\star}}{\delta} \int_{s}^{s+\Delta} 2 V(\tau)|A(\tau)| d \tau .
$$

Similarly the term $I_{4}$ satisfies the bound

$$
\left|I_{4}\right| \leq \int_{s}^{s+\Delta}\left|\bar{e}^{T}(\tau)\right|^{2} \frac{1}{\left|\bar{e}^{T}(\tau)\right|}|\dot{r}(\tau)| d \tau,
$$

which is, for all $|e|>\delta$ and taking into account the bound on $|\dot{r}(t)|$, bounded by

$$
\left|I_{4}\right| \leq \frac{\rho_{\star}}{\delta} \int_{s}^{s+\Delta} 2 V(\tau) d \tau .
$$

Combining (33), (34), (35), (37) and (39), for all $|\bar{e}|>\delta$ we obtain

$$
\begin{aligned}
& V(s+\Delta) \leq\left(\frac{2 \Delta b_{\star}\left(\sqrt{a_{\star}} r_{\star}+\rho_{\star}\right)(1-\gamma)}{\delta^{2} \beta_{0}}+\gamma\right) V(s) \\
& +\int_{s}^{s+\Delta}\left(2|A(\tau)|\left(1+\frac{r_{\star}}{\delta}\right)+\frac{2 \rho_{\star}}{\delta}+\frac{a_{\star}}{\Delta} \gamma_{2}\right) V(\tau) d \tau,
\end{aligned}
$$

where $\gamma$ and $\gamma_{2}$ are defined in (25) and (26) respectively. Noting that

$$
\frac{k \alpha \Delta \beta_{0}}{1+2 k^{2} \alpha^{2} \Delta^{2} b_{\star}^{2}} \leq \frac{\beta_{0}}{2 \sqrt{2} b_{\star}}
$$

and that $\beta_{0} \leq b_{\star}$, we get that $\gamma \in\left(\frac{2 \sqrt{2}-1}{2 \sqrt{2}}, 1\right)$, which implies that $\gamma$ is positive. We now apply the Bellman-Gronwall lemma, and get that for all $s \geq T$, for all $|e|>\delta$,

$$
V(s+\Delta) \leq \gamma_{3} e^{\int_{s}^{s+\Delta}\left(2|A(\tau)|\left(1+\frac{r_{\star}}{\delta}\right)+\frac{2 \rho_{\star}}{\delta}+\frac{a_{\star}}{\Delta} \gamma_{2}\right) d \tau} V(s),
$$

Where $\gamma_{3}$ is as defined in (27). We note that the CauchySchwartz inequality yields $\int_{s}^{s+\Delta}|A(\tau)| d \tau \leq \Delta \sqrt{a_{\star}}$, so we get, for all $s \geq T$,

$$
V(s+\Delta) \leq \gamma_{3} e^{\left(2 \Delta\left[\sqrt{a_{\star}}\left(1+\frac{r_{\star}}{\delta}\right)+\frac{\rho_{\star}}{\delta}\right]+a_{\star} \gamma_{2}\right)} V(s),
$$

where $\gamma_{2}$ is as defined in (26). Evidently for convergence we require that

$$
\gamma_{3}<e^{-\left(2 \Delta\left[\sqrt{a_{\star}}\left(1+\frac{r_{\star}}{\delta}\right)+\frac{\rho_{\star}}{\delta}\right]+a_{\star} \gamma_{2}\right)} .
$$

The left side of inequaity (42), $\gamma_{3}$, is strictly increasing as a function of $a_{\star}$. The exponential on the right side is strictly decreasing. Therefore if equality holds in (42) for some $\bar{a}_{\star}$ then inequality (42) is guaranteed to hold for all $a_{\star} \in\left(0, \bar{a}_{\star}\right)$, which by application of Theorem 1 proves that the origin of system (22), (23) is $\left(\frac{1}{\omega}, \delta\right)$-SPUUB, proving the theorem under condition (i). To study the convergence rate of our system we define

$$
\gamma_{r}=\gamma_{3} e^{\left(2 \Delta\left[\sqrt{a_{\star}}\left(1+\frac{r_{\star}}{\delta}\right)+\frac{\rho_{\star}}{\delta}\right]+a_{\star} \gamma_{2}\right)}<1 .
$$

For any $t \geq T$ we denote $N=\left\lfloor\frac{t-T}{\Delta}\right\rfloor$, where $\lfloor\cdot\rfloor$ is the floor function. Then for $t \geq T$ we have $t=T+$ $\Delta\left(\frac{t-T}{\Delta}-\left\lfloor\frac{t-T}{\Delta}\right\rfloor\right)+N \Delta$ and from (41) we have the bound

$$
V(t) \leq \gamma_{r}^{N} V\left(T+\Delta\left(\frac{t-T}{\Delta}-\left\lfloor\frac{t-T}{\Delta}\right\rfloor\right)\right)
$$

This bound is obtained by noting from (41) and (43) that $V(s+N \Delta) \leq \gamma_{r}^{N} V(s)$ and by substituting $s=T+$ $\Delta\left(\frac{t-T}{\Delta}-\left\lfloor\frac{t-T}{\Delta}\right\rfloor\right)$. Recalling that for $|e|>\delta$,

$$
\begin{aligned}
\dot{V} & =\bar{e}^{T}\left[A(t)-k \alpha B(t) B^{T}(t)\right] \bar{e}+\bar{e}^{T} A(t) r-\bar{e}^{T} \dot{r} \\
& \leq 2\left(\left|A(t)-k \alpha B(t) B^{T}(t)\right|+\frac{|A(t) r|+|\dot{r}|}{\delta}\right) V(t),
\end{aligned}
$$

we define

$$
M_{1}=e^{\int_{0}^{T+\Delta} 2\left(\left|A(\tau)-k \alpha B(\tau) B^{T}(\tau)\right|+\frac{|A(\tau) r(\tau)|+|\dot{r}(\tau)|}{\delta}\right) d \tau},
$$

and because $\Delta\left(\frac{t-T}{\Delta}-\left\lfloor\frac{t-T}{\Delta}\right\rfloor\right) \leq \Delta$ we get the bound

$$
V\left(T+\Delta\left(\frac{t-T}{\Delta}-\left\lfloor\frac{t-T}{\Delta}\right\rfloor\right)\right) \leq M_{1} V(0) .
$$

Therefore

$$
V(t) \leq M_{1} \gamma_{r}^{N} V(0)
$$

We now consider the term $\gamma_{r}^{N}$. Since $N=$ $\frac{t-T-\Delta\left(\frac{t-T}{\Delta}-\left\lfloor\frac{t-T}{\Delta}\right\rfloor\right)}{\Delta} \geq \frac{t-T-\Delta}{\Delta}$, and $\gamma_{r} \in(0,1)$ it follows that

$$
\gamma_{r}^{N} \leq \gamma_{r}^{\frac{t-T-\Delta}{\Delta}}
$$

With (45) and (46) we obtain

$$
V(t) \leq M_{1} \gamma_{r}^{-\frac{T+\Delta}{\Delta}} \gamma_{r}^{\frac{t}{\Delta}} V(0) .
$$

We now define $M=\sqrt{M_{1} \gamma_{r}^{-\frac{T+\Delta}{\Delta}}}$ and rewrite $\gamma_{r}^{\frac{t}{\Delta}}=$ $\left(\frac{1}{\gamma_{r}}\right)^{\frac{-t}{\Delta}}=e^{-\frac{\ln \left(\frac{1}{\gamma_{r}}\right)}{\Delta} t}$. Recalling that $\gamma_{r} \in(0,1)$ we define

$$
R\left(k \alpha, \Delta, \beta_{0}, b_{\star}, a_{\star}\right)=\frac{\ln \left(\frac{1}{\gamma_{r}}\right)}{2 \Delta}>0,
$$

and write the exponential decay of $V$ as

$$
V(t) \leq M^{2} e^{-2 R t} V(0)
$$

Substituting (43) into (48), we obtain (24). Finally recalling the definition of $V(t)$ we write the exponential decay of $|\bar{e}(t)|$ as

$$
|\bar{e}(t)| \leq M e^{-R t}|\bar{x}(0)| .
$$

Therefore $\bar{e}$ exponentially converges to within a $\delta$-ball of the origin with average convergence rate $\mathrm{R}$ as in (24). Proceeding to the proof of the theorem under condition (ii), for any given $a_{\star}$ we want to find a range of stabilizing values of $k \alpha$ as a function of $\Delta$. For a given $\beta_{0}, b_{\star}, a_{\star}$ we first consider over what range of $\Delta \in(0, \infty)$ it is possible to satisfy the convergence condition (42). We define the function

$$
\begin{aligned}
F(k \alpha, \Delta)= & \frac{k \alpha \Delta \beta_{0}}{1+2 k^{2} \alpha^{2} \Delta^{2} b_{\star}^{2}}\left[1-\frac{2 \Delta b_{\star}\left(\sqrt{a_{\star}} r_{\star}+\rho_{\star}\right)}{\delta^{2} \beta_{0}}\right] \\
& +e^{-\left(2 \Delta\left[\sqrt{a_{\star}}\left(1+\frac{r_{\star}}{\delta}\right)+\frac{\rho_{\star}}{\delta}\right]+a_{\star} \gamma_{2}\right)}
\end{aligned}
$$

which must have a value greater than 1 for (42) to be satisfied. In order to calculate the maximum possible value of (51) we fix $\Delta$ and set the derivative, with respect to $k \alpha$, of $F(k \alpha, \Delta)$ equal to zero, to find that $F(k \alpha, \Delta)$ has its maximum value at $(k \alpha)_{\mathrm{m}}=\frac{1}{\sqrt{2} \Delta b_{\star}}$ and the maximum value 
is

$$
\begin{aligned}
F\left((k \alpha)_{\mathrm{m}}, \Delta\right)= & \frac{\beta_{0}}{2 \sqrt{2} b_{\star}}\left[1-\frac{2 \Delta b_{\star}\left(\sqrt{a_{\star}} r_{\star}+\rho_{\star}\right)}{\delta^{2} \beta_{0}}\right] \\
& +e^{-\left(\sqrt{2} \Delta^{2} a_{\star}+2 \Delta \sqrt{a_{\star}}\left[1+\frac{r_{\star}}{\delta}\right]+\frac{2 \rho_{\star} \Delta}{\delta}\right)} .
\end{aligned}
$$

The convergence condition requires this maximum value (52) to be greater than 1 . We note that $F\left((k \alpha)_{\mathrm{m}}, \Delta\right)$ is strictly decreasing as a function of $\Delta \in(0, \infty)$. Therefore if $F\left((k \alpha)_{\mathrm{m}}, \bar{\Delta}_{1}\right)=1$, it follows that $F\left((k \alpha)_{\mathrm{m}}, \Delta\right)>1$ for all $\Delta \in\left(0, \bar{\Delta}_{1}\right)$. The condition $F\left((k \alpha)_{\mathrm{m}}, \bar{\Delta}_{1}\right)=1$ implies that

$$
\begin{aligned}
0= & \bar{\Delta}_{1}^{2} \sqrt{2} a_{\star}+\bar{\Delta}_{1} 2\left(\sqrt{a_{\star}}\left[1+\frac{r_{\star}}{\delta}\right]+\frac{\rho_{\star}}{\delta}\right) \\
& +\ln \left(1+\frac{\beta_{0}}{2 \sqrt{2} b_{\star}}\left[\frac{2 \bar{\Delta}_{1} b_{\star}\left(\sqrt{a_{\star}} r_{\star}+\rho_{\star}\right)}{\delta^{2} \beta_{0}}-1\right]\right) .
\end{aligned}
$$

Therefore it is possible to stabilize the system when $0<$ $\Delta<\bar{\Delta}_{1}$ by choosing $k \alpha=(k \alpha)_{\mathrm{m}}=\frac{1}{\sqrt{2} \Delta b_{\star}}$. By continuity, for any $0<\Delta<\bar{\Delta}_{1}$ there must be an interval containing $(k \alpha)_{\mathrm{m}}$ such that all values of $k \alpha$ within that interval satisfy condition (42). For $\Delta \in\left(0, \bar{\Delta}_{1}\right)$ we consider all values of $k \alpha$ that achieve $F(k \alpha, \Delta)>1$. Recalling that

$$
\begin{aligned}
F(k \alpha, \Delta)= & \frac{k \alpha \Delta \beta_{0}}{1+2 k^{2} \alpha^{2} \Delta^{2} b_{\star}^{2}}\left[1-\frac{2 \Delta b_{\star}\left(\sqrt{a_{\star}} r_{\star}+\rho_{\star}\right)}{\delta^{2} \beta_{0}}\right] \\
& +e^{-\left(2 \Delta\left[\sqrt{a_{\star}}\left(1+\frac{r_{\star}}{\delta}\right)+\frac{\rho_{\star}}{\delta}\right]+a_{\star} \gamma_{2}\right)},
\end{aligned}
$$

to remove the $k \alpha$ dependence from the exponential in (54) we write out $\gamma_{2}$ and restrict our attention to $k \alpha>1$, in which case

$$
e^{-2 \Delta\left(\frac{\sqrt{a_{\star} \delta+r_{\star}+\rho_{\star}}}{\delta}+\frac{2 k \alpha \Delta^{2} b_{\star} a_{\star}}{1+2 k^{2} \alpha^{2} \Delta^{2} b_{\star}^{2}}\right)}>e^{-2 \Delta\left(\frac{\sqrt{a_{\star} \delta+r_{\star}+\rho_{\star}}}{\delta}+\frac{a_{\star}}{b_{\star}}\right) .}
$$

We satisfy $F(k \alpha, \Delta)>1$ by restricting $k \alpha$ to satisfy

$$
\begin{aligned}
& \frac{k \alpha \Delta \beta_{0}}{1+2 k^{2} \alpha^{2} \Delta^{2} b_{\star}^{2}}\left[1-\frac{2 \Delta b_{\star}\left(\sqrt{a_{\star}} r_{\star}+\rho_{\star}\right)}{\delta^{2} \beta_{0}}\right] \\
& +e^{-2 \Delta\left(\frac{\sqrt{a_{\star} \delta+r_{\star}+\rho_{\star}}}{\delta}+\frac{a_{\star}}{b_{\star}}\right)}>1 .
\end{aligned}
$$

Setting (56) equal to 1 , we solve for $k \alpha$ as

$$
\begin{aligned}
& k \alpha=\frac{\beta_{0}\left[\frac{1-\gamma_{3}}{1-\gamma}\right]}{4 \Delta b_{\star}^{2}\left[1-e^{\left.-2 \Delta\left(\frac{\sqrt{a_{\star} \delta+r_{\star}+\rho_{\star}}}{\delta}+\frac{a_{\star}}{b_{\star}}\right)\right]}\right.} \\
& \pm \frac{\sqrt{\beta_{0}^{2}\left[\frac{1-\gamma_{3}}{1-\gamma}\right]^{2}-8 b_{\star}^{2}\left[1-e^{\left.-2 \Delta\left(\frac{\sqrt{a_{\star} \delta+r_{\star}+\rho_{\star}}}{\delta}+\frac{a_{\star}}{b_{\star}}\right)\right]^{2}}\right.}}{4 \Delta b_{\star}^{2}\left[1-e^{\left.-2 \Delta\left(\frac{\sqrt{a_{\star} \delta+r_{\star}+\rho_{\star}}}{\delta}+\frac{a_{\star}}{b_{\star}}\right)\right]}\right.}
\end{aligned}
$$

To ensure $k \alpha$ is real valued we impose the condition

$$
\begin{aligned}
\beta_{0}^{2}\left[\frac{1-\gamma_{3}}{1-\gamma}\right]^{2} & =\beta_{0}^{2}\left[1-\frac{2 \Delta b_{\star}\left(\sqrt{a_{\star}} r_{\star}+\rho_{\star}\right)}{\delta^{2} \beta_{0}}\right]^{2} \\
& \geq 8 b_{\star}^{2}\left[1-e^{-2 \Delta\left(\frac{\sqrt{a_{\star} \delta+r_{\star}+\rho_{\star}}}{\delta}+\frac{a_{\star}}{b_{\star}}\right)}\right]^{2} .
\end{aligned}
$$

Note that the left side of (58) is strictly increasing while the right side is strictly decreasing as $\Delta$ decreases. Therefore if equality in (58) holds for some $\bar{\Delta}_{2}$, then for all $\Delta<\bar{\Delta}_{2}$ the inequality will hold. Therefore (58) holds for all $0<\Delta<$ $\bar{\Delta}_{2}$ where $\bar{\Delta}_{2}$ satisfies

$$
\begin{aligned}
& \beta_{0} \delta^{2}-2 \bar{\Delta}_{2} b_{\star}\left(\sqrt{a_{\star}} r_{\star}+\rho_{\star}\right) \\
& =2 \sqrt{2} b_{\star} \delta^{2}\left[1-e^{-2 \Delta\left(\frac{\sqrt{a_{\star}} \delta+r_{\star}+\rho_{\star}}{\delta}+\frac{a_{\star}}{b_{\star}}\right)}\right],
\end{aligned}
$$

which implies that the new requirement on the possible values of $\Delta$ is $0<\Delta<\min \left\{\bar{\Delta}_{1}, \bar{\Delta}_{2}\right\}$, from which we obtain (29), (30). Returning to (57) and recalling the value $(k \alpha)_{\mathrm{m}}=\frac{1}{\sqrt{2} \Delta b_{\star}}$ we have the roots $k \alpha=\frac{(k \alpha)_{\mathrm{m}}}{\eta}$ and $k \alpha=$ $(k \alpha)_{\mathrm{m}} \eta$, where

$$
\begin{aligned}
\eta= & \frac{\beta_{0}\left[\frac{1-\gamma_{3}}{1-\gamma}\right]}{2 \sqrt{2} b_{\star}\left[1-e^{\left.-2 \Delta\left(\frac{\sqrt{a_{\star} \delta+r_{\star}+\rho_{\star}}}{\delta}+\frac{a_{\star}}{b_{\star}}\right)\right]}\right.} \\
& +\frac{\sqrt{\beta_{0}^{2}\left[\frac{1-\gamma_{3}}{1-\gamma}\right]^{2}-8 b_{\star}^{2}\left[1-e^{\left.-2 \Delta\left(\frac{\sqrt{a_{\star} \delta+r_{\star}+\rho_{\star}}}{\delta}+\frac{a_{\star}}{b_{\star}}\right)\right]^{2}}\right.}}{2 \sqrt{2} b_{\star}\left[1-e^{\left.-2 \Delta\left(\frac{\sqrt{a_{\star} \delta+r_{\star}+\rho_{\star}}}{\delta}+\frac{a_{\star}}{b_{\star}}\right)\right]}\right.} .
\end{aligned}
$$

Therefore the system is stable for

$$
k \alpha \in\left(\frac{(k \alpha)_{\mathrm{m}}}{\eta},(k \alpha)_{\mathrm{m}} \eta\right) .
$$

We have thus derived sufficient conditions on $\Delta$ and $k \alpha$ to guarantee stability of our system. For each window $\Delta$ we have given an interval of stabilizing values of $k \alpha,(60)$. However we now restrict our conditions on $k \alpha$ in order to give a more intuitive condition (31). We show that the interval (60) contains $(k \alpha)_{\mathrm{m}}$ by recalling (58) and verifying that $\eta \geq 1$ and therefore $\frac{1}{\eta}<1$. Therefore the interval (60) contains the more restrictive, but more illustrative interval (31), where we have explicitly written out the value $(k \alpha)_{\mathrm{m}}=$ $\frac{1}{\sqrt{2} \Delta b_{\star}}$. From the presence of $1-e^{-\Delta(\ldots)}$ in the denominator we see that this interval of stability grows unbounded in length as the window $\Delta$ decreases.

The nonlinear extension of our tracking results, stated without proof is a trivial modification of [1, Theorem 5].

\section{Theorem 3: Consider the nonlinear system}

$$
\dot{x}=f(x, t)+G(x, t) u,
$$

where $f, u: \mathbb{R}^{n} \times \mathbb{R} \rightarrow \mathbb{R}^{n}, G: \mathbb{R}^{n \times n} \times \mathbb{R} \rightarrow \mathbb{R}^{n \times n}$, with $f$ and $G$ each having separable dependence on $x$ and $t$. Let there exist $\eta \in \mathcal{K}_{\infty}$ and $\beta_{0}>0$ such that $f$ and $G$ satisfy the following bounds for all $t \in \mathbb{R}^{+}, x \in \mathbb{R}^{n}$ :

$$
G(x, \tau) G^{T}(x, \tau) \geq \beta_{0} I,|f(x, t)| \leq \eta(|x|) .
$$

Consider the error system

$$
\dot{e}=f(e, t)+G(e, t) u-\dot{r},
$$


under the influence of the controller

$$
u_{i}=\alpha \sqrt{\omega \omega_{i}^{\prime}} \cos \left(\omega \omega_{i}^{\prime} t\right)-k \sqrt{\omega \omega_{i}^{\prime}} \sin \left(\omega \omega_{i}^{\prime} t\right) \int_{0}^{|e|} \eta(r) d r,
$$

where the frequencies $\omega_{i}^{\prime}$ are rational and distinct. If $k \alpha$ is chosen such that

$$
k \alpha>\frac{1}{\beta_{0}}+\frac{\rho_{\star}}{\beta_{0} \eta(\delta)}
$$

then origin of (62), (63) is $\left(\frac{1}{\omega}, \delta\right)$-SPUUB.

\section{Simulation Results}

To demonstrate the extremum seeking controller's ability to handle unknown, quickly time varying control direction we consider the system

$$
\left[\begin{array}{l}
\dot{x}_{1} \\
\dot{x}_{2}
\end{array}\right]=\left[\begin{array}{cc}
1.1 & 1.2 \\
-1.1 & 1
\end{array}\right]\left[\begin{array}{l}
x_{1} \\
x_{2}
\end{array}\right]+\left[\begin{array}{l}
\cos (20 t+.3) \\
\sin (20 t+.3)
\end{array}\right] u
$$

which we want to follow the trajectory

$$
r(t)=[\cos (t / 4), \sin (t / 2)]^{T} .
$$

A physical motivation for this example can be that $x=$ $\left(x_{1}, x_{2}\right)$ is the planar coordinate of a mobile robot, with its angular velocity actuator failed and stuck at 20, and which needs to follow the trajectory $r(t)$ using the forward velocity input $u$ only, in the presence of a position-dependent perturbation given by $\left[\begin{array}{cc}1.1 & 1.2 \\ -1.1 & 1\end{array}\right] x$. The uncontrolled system is unstable with poles at $1.05 \pm 1.15 i$. We apply ES control

$$
u=\alpha \sqrt{\omega} \cos (\omega t)-k \sqrt{\omega} \sin (\omega t)|e(t)|^{2},
$$

with parameteres $\omega=400, k=10$, and $\alpha=3$ and start from $x_{1}(0)=1, x_{2}(0)=-1$. Figure 1 shows the system's trajectory over 26 seconds. Following an initial transient the control effort settles to a periodic slightly amplitude modulated waveform whose magnitude depends on $|e|$, also shown in Figure 1.

\section{CONCLUSions}

The extremum seeking algorithm creates a closed loop system that is independent of the control vector's direction. This is a useful property which allows us to stabilize and perform trajectory tracking with unknown, unstable, control direction-varying systems using a particular form of timevarying nonlinear high-gain feedback. In the LTV case the only restriction to the applicability of the control law (1) is that, for a given bound on $A(t)$, the vector $B(t)$ be persistently exciting over a sufficiently short window $\Delta$, namely, that the variations of $B(t)$ are sufficiently fast. In the nonlinear case we require that the control vector $G(x, t)$ is non-zero. In both cases we achieve semiglobal uniform ultimate boundedness of the error system, with ultimate bound $\delta>0$.

\section{REFERENCES}

[1] A. Scheinker and M. Krstic, "A universal extremum seeking-based stabilizer for unknown LTV systems with unknown control directions," Proc. ACC, Montreal, Canada, 2012.
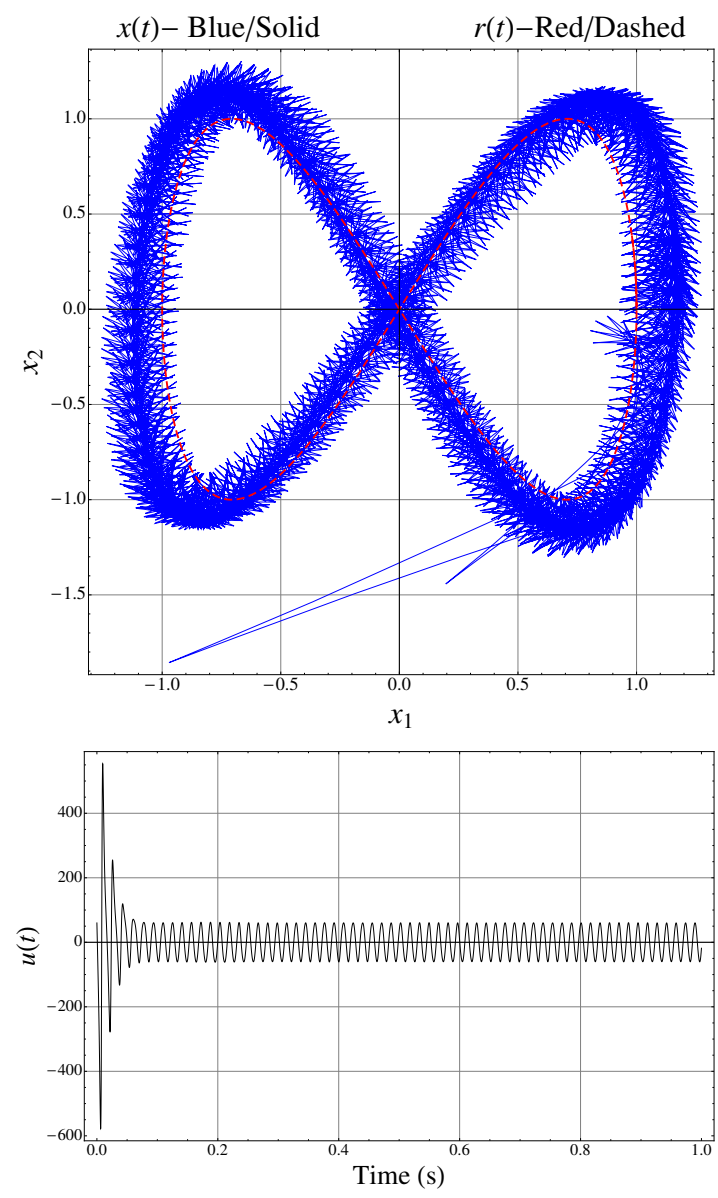

Fig. 1. As the system trajectory approaches $r(t)$ the control effort quickly settles to an almost periodic waveform with amplitude modulation which is due to the fact that the disturbing term $A x$ has magnitude which depends on position.

[2] H. Dürr, M. Stanković, K. Johansson, "A Lie bracket approximation for extremum seeking vehicles." Proc. IFAC World Cong., Milan, 2011.

[3] L. Gurvits, "Averaging approach to nonholonomic motion planning," Proc. IEEE. Conf. Robotics and Automation, Nice, France, May 1992

[4] L. Gurvits and Z. Li, "Smooth time-periodic solutions for nonholonomic motion planning," in Z. Li and J.F. Canny., Eds., Nonholonomic Motion Planning, Kluwer, 1992.

[5] P. Ioannou and J. Sun, Robust Adaptive Control, Prentice Hall, 1996.

[6] M. Krstic and H. Wang, "Stability of extremum seeking feedback for general dynamic systems." Automatica, vol. 36, pp. 595-601, 2000.

[7] L. Moreau and D. Aeyels, "Practical stability and stabilization." IEEE Trans. Automat. Contr., vol. 45, pp. 1554-1558, 2000.

[8] D. Mudgett and S. Morse, "Adaptive stabilization of linear systems with unknown high-frequency gains." IEEE Trans. Automat. Contr., vol. 30, pp. 549-554, 1985.

[9] Y. Tan, D. Nešić, and I. Mareels, "On non-local stability properties of extremum seeking control," Automatica, vol. 42, pp. 889-903, 2006.

[10] C. Zhang, A. Siranosian, and M. Krstic, "Extremum seeking for moderately unstable systems and for autonomous vehicle target tracking without position measurements, Automatica, vol. 43, pp. 1832-1839, 2007

[11] M. Krstic and H. Deng, Stabilization of Nonlinear Uncertain Systems. NY, Springer-Verlag, 1998.

[12] V. Arnold, Mathematical Methods of Classical Mechanics. Moscow, Nauka, 1974

[13] H. Dürr, M. Stanković, K. Johansson, (2011, Nov 17) "Nash Equilibrium Seeking in Multi-Vehicle Systems: A Lie Bracket Approximation-Based Approach." [Online]. Available: http://arxiv.org/abs/1109.6129 